\title{
Comparing Accuracy of Cerebral Aneurysm Size Measurements From Three Routine Investigations: Computed Tomography, Magnetic Resonance Imaging, and Digital Subtraction Angiography
}

\author{
Hiroyuki TAKAO, Yuichi MURAYAMA, Toshihiro ISHIBASHI, \\ Takayuki SAGUCHI, Masaki EBARA, Hideki ARAKAWA, Koreaki IRIE, \\ Kiyotaka IWASAKI*, Mitsuo UMEZU*, and Toshiaki ABE \\ Division of Endovascular Neurosurgery and Neurosurgery, \\ Jikei University School of Medicine, Tokyo; \\ * Major in Mechanical Engineering \& Major in Integrative Bioscience and Biomedical Engineering, \\ Graduate School of Science and Engineering, Waseda University, Tokyo
}

\begin{abstract}
Modern imaging technologies, such as computed tomography (CT) angiography, magnetic resonance (MR) angiography, and digital subtraction (DS) angiography are widely used for pretreatment evaluation of cerebral aneurysms, but the relative accuracies of these modalities are unclear. This study compared the measurements of aneurysm neck and dome height and width on CT angiography, time-offlight (TOF)-MR angiography, and DS angiography using a three-dimensional workstation. An elastic model of a side-wall aneurysm was connected to an artificial heart pulsatile circuit system. The aneurysm model was prepared using a silicone membrane of 0.6-mm thickness under normal physiological circulation parameters. Using this aneurysm model, three-dimensional TOF-MR angiography, contrast-enhanced CT angiography, and DS angiography were performed. Source images were postprocessed on a dedicated workstation to calculate the aneurysm size. DS angiography measurements were found to be the most accurate. In contrast, aneurysm neck sizes measured on CT angiography were significantly wider than actual values $(p<0.05)$ and aneurysm heights measured using TOF-MR angiography were significantly lower than actual values $(p<0.01)$. In this in-vitro model, at least one aneurysm dimension measured with CT angiography and with TOF-MR angiography differed significantly from actual values. Aneurysm neck width markedly affects therapeutic planning, as a wide neck requires craniotomy or endovascular treatment using an adjunctive device, so inaccuracies should be considered when aneurysm treatment is planned using modern methods of visualization.
\end{abstract}

Key words: cerebral aneurysm, size measurement, computed tomography, magnetic resonance imaging, digital subtraction angiography

\section{Introduction}

Non-invasive imaging modalities such as magnetic resonance (MR) angiography and three-dimensional (3D) computed tomography (CT) angiography are being increasingly used for the detection and evaluation of cerebral aneurysms. ${ }^{6,10,12,18,20,21,25)}$ One recent study found that the sensitivities of CT and MR angiography for preoperative evaluation of cerebral aneurysms larger than $3 \mathrm{~mm}$ in diameter were $96 \%$

Received September 28, 2009; Accepted March 29, 2010 and $94 \%$, respectively, and $61 \%$ and $38 \%$ for aneurysms smaller than $3 \mathrm{~mm}$ in diameter, respectively, and specificity was $90 \%$ for both size ranges. ${ }^{22)}$ With improvements in CT angiography, some institutions have started to perform surgical clipping on the basis of only preoperative 3D-CT angiography, rather than digital subtraction (DS) angiography. ${ }^{12)}$ Technical improvements have likewise enabled time-of-flight (TOF)-MR angiography to produce images comparable in quality to 3D-DS angiography without the use of contrast media. Some institutions have even attempted to perform surgery using only MR angiography. ${ }^{18,21)}$ Such institutions 
may also have to determine whether endovascular coil embolization is indicated on the basis of 3D-CT or MR angiography findings.

Accurate determination of the aneurysm neck size is critical for planning endovascular coil embolization. Wide-necked aneurysms are considered to be difficult to treat with only coil embolization and are better treated in combination with intracranial stents or balloons. Although many factors affect the obliteration rate, the most important is the dome to neck ratio. ${ }^{4,14)}$ However, the obtained measurements of the cerebral aneurysm may vary according to the method (MR, DS, or CT angiography) used. The accuracy of various imaging modalities has been examined previously, ${ }^{17,24)}$ but few reports have compared different imaging modalities on the same 3D workstation.

The present study performed in-vitro measurements using a silicone pulsatile cerebral aneurysm model to assess the accuracy of measuring aneurysm size and neck width with CT, TOF-MR and DS angiography on a dedicated 3D workstation.

\section{Methods}

A silicone aneurysm model was used to approximate the elasticity of cerebral aneurysms (Fig. 1). A sidewall model was used, similar to an aneurysm of the internal carotid artery. The reported arterial stiffness parameter $\beta$ for the internal carotid artery is $10.4 \pm 4.8 .^{7,9,15)}$ Therefore, the $\beta$ of the model vessel was set at 10 to match that of the internal carotid artery. The aneurysm was designed with a dome of 14 $\mathrm{mm}$ in diameter and a neck of $5 \mathrm{~mm}$ width. The membrane thickness in the aneurysm model was 0.6 $\mathrm{mm}$, which is the minimum thickness of silicone that approximated the elasticity of the human carotid artery within our technical possibilities.

The model section was set up by branching off a circulation circuit consisting of a pressure-driven artificial heart pulsatile pump (Spiral Vortex Pump; Waseda University, Tokyo), compliance tank, resistor, and reservoir tank (Fig. 2). The pulse rate was fixed at 60 beats/min. Blood pressure was set as $120 / 80 \mathrm{mmHg}$. The pulsatile circulation simulator and pressure flow waveforms in the common carotid artery of the body are shown in Fig. 3. The abrupt rise in the contraction phase of flow waveforms, which is closely related to the blood flow in the aneurysm in the pulsatile circulation simulation, was similar to that in the physiological arterial waveforms. In addition, mean flow was set at $300 \mathrm{ml} / \mathrm{min}$ on the basis of the percentage of blood in the common carotid artery that enters the internal carotid artery $(60 \%)$ and, subsequently, the cerebral

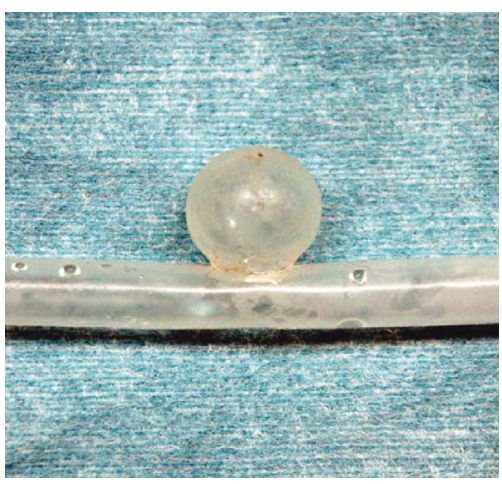

Fig. 1 Photograph of the silicone cerebral aneurysm model made of silicone to simulate the vascular elasticity of the internal carotid artery: thickness, $0.6 \mathrm{~mm}$; aspect ratio, 2.5; and vascular stiffness parameter $(\beta)$, 10.

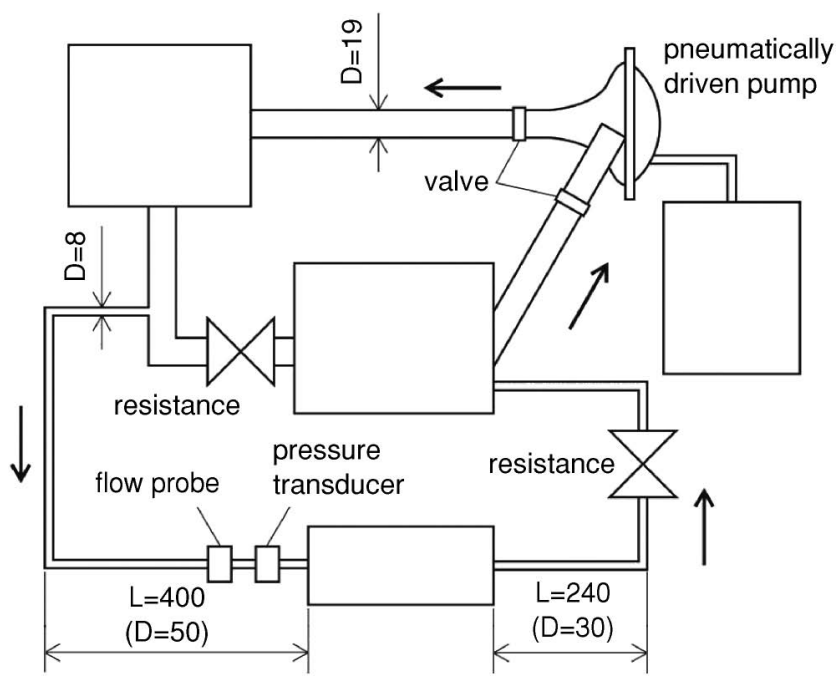

Fig. 2 Schematic of the in vitro pulsatile simulator. D: diameter (mm), L: length (mm).

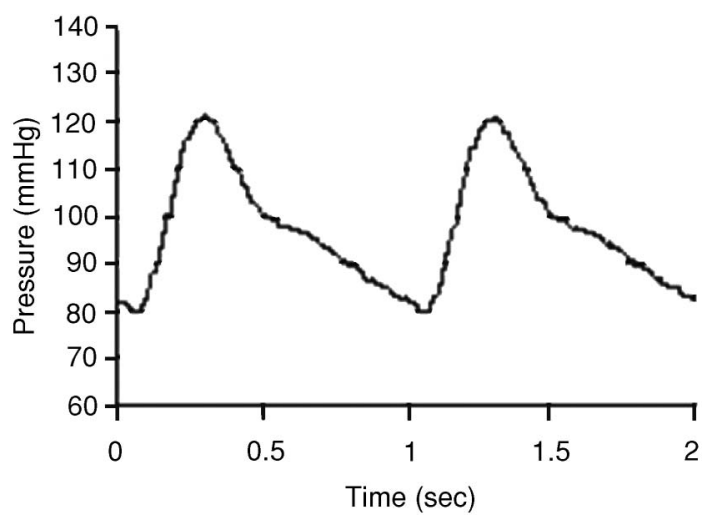

Fig. 3 Typical pressure and flow waveforms in the "internal carotid artery" of the simulator. 
arteries. ${ }^{19)}$ The working fluid was a Newtonian blood-analog fluid consisting of a mixture of $46 \%$ glycerol and $54 \%$ water by volume. The corresponding mean Reynolds and Womersley numbers of the mixture were 270 and 3.8, respectively. For the CT angiography test, $10 \%$ of the volume was replaced by contrast medium (Omnipaque 300; Daiichi Sankyo Co. Ltd, Tokyo), and for the DS angiography, $40 \%$ was replaced.

The silicone model changes size with pulsation, so the dimensions were measured during the pulsatile simulation. These measurements were performed on video footage of the model pulsation under digital microscopy. Images of both contraction and expansion phases were chosen from the footage and analyzed using VH Analyzer software (Keyence, Osaka). Internal measurements independently performed by three technicians included height, width, and neck size of the silicone aneurysm model, as the circulating fluid was dyed with red ink. Mean height was $14.35 \pm 0.04 \mathrm{~mm}$, mean width was $13.59 \pm 0.01$ $\mathrm{mm}$, and mean neck size was $4.83 \pm 0.10 \mathrm{~mm}$. These dimensions were considered as the real size for comparison with values from the different diagnostic modalities.

3D-MR angiography was performed using a 1.5-T Magnetom Symphony system (Siemens, Erlangen, Germany). The aneurysm model was placed horizontally so that the vessel ran parallel to the scanner table with the aneurysm perpendicular to the long axis of the table. Using a TOF sequence, the scan protocol was: repetition time (TR), $37 \mathrm{msec}$; echo time, $7.15 \mathrm{msec}$; flip angle, $25^{\circ}$; scan time, $6 \mathrm{~min}$; matrix, $256 \times 256$; field-of-view (FOV), $215 \mathrm{~mm}$; slice thickness, $0.9 \mathrm{~mm}$; and number of slices, 96 (3 slabs). Images were displayed as cross sections using maximum intensity projection (MIP) to include the parent vessel and the center of the aneurysm, followed by determining distances between points, using the distance measurement function.

3D-CT angiography used a Somatom Sensation 16slice CT system (Siemens). The aneurysm model was placed horizontally so that the vessel ran parallel to the table and the aneurysm perpendicular to the long axis of the table. Scan parameters comprised: rotation period, $0.5 \mathrm{sec}$; tube voltage, $120 \mathrm{kV}$; tube current, $250 \mathrm{~mA}$; beam collimation, $16 \times 0.75 \mathrm{~mm}$; pitch factor, 0.667; slice thickness, $0.75 \mathrm{~mm}$; matrix, $512 \times 512$; FOV, $74 \mathrm{~mm}$; and standard function for H50 head measurement. Reconstructed images were displayed as cross sections using a 3-mm-thick slab MIP to include the parent vessel and center points of the aneurysm. Distances between points were then determined with the distance measurement function.
DS angiography was performed using the AXIOM Artis dBA system (Siemens). The aneurysm model was positioned as for the CT and MR angiography measurements. The images were taken with a vertical x-ray beam (similar to anteroposterior). Angiographic series parameters comprised: tube voltage, $81 \mathrm{kV}$; tube current, $133 \mathrm{~mA}$; frame rate, 7.5 frames/sec; FOV, $130 \mathrm{~mm}$; and matrix, $1024 \times 1024$. Distances between points were measured from the obtained image using the previously calibrated distance-measurement function. To assess cerebral aneurysm dimensions, the same distances as pretreatment CT angiography or TOF MIP MR angiography were measured on the two-dimensional (2D) DS angiogram. No 3D-DS angiography was performed.

In the present study, the reference values were the actual measurements made on the silicone model. When distances are measured on cross-sections, values at the window center influence aneurysm measurements. ${ }^{2)}$ Therefore, an optimized window set-up considering the image properties of each equipment was standardized for the measurements. For CT angiography (MIP) and DS angiography, the density scale on images is almost proportional to the $\mathrm{x}$-ray dosage, ${ }^{1,8)}$ so the value midway between the maximum density of the aneurysm and the background density around the aneurysm was considered to represent the window center value. For MR angiography, density scale (signal strength) is not linear against tissue. ${ }^{11)}$ The TOF scan sequence used for MR angiography represents flow of liquid rather than density. Therefore, the median value between minimum density, at which the outline of the aneurysm blends with the noise of the surrounding area and expands unevenly, and maximum density, at which the outline shrinks unevenly due to noise inside the aneurysm area, was considered to represent the window center value.

Source images were post-processed on the same dedicated syngo ${ }$ 3D workstation (Siemens) to calculate the aneurysm size. The aneurysm neck was defined as the vascular surface plane of the affected vessel with access to the aneurysm cavity. The aneurysm height was defined as the distance between the surface plane and the aneurysm fundus tip, and the aneurysm width was defined as the maximum horizontal length of the aneurysm (parallel to the neck plane) (Fig. 4). On the workstation, the aneurysm neck, height, and width were measured on the aneurysm model. For actual measurements, mean maximum and minimum values were determined during scanning, and standard deviations were obtained. Scanning was performed 3 times for each modality under the same conditions using the 
same workstation. Measurements were performed in triplicate for each of the 3 modalities by 2 independent radiology technicians, meaning that 9 measurements were performed by each technician. For CT and MR angiography, both mean and standard deviations were calculated. For DS angiography, mean maximum and minimum values

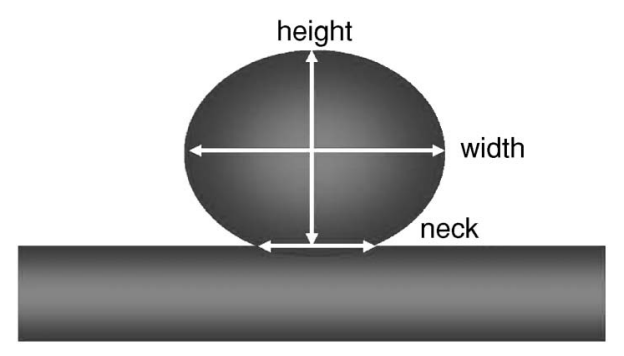

Fig. 4 Diagram showing the aneurysm measurements. and standard deviations (of pulsatile change) were obtained during scanning.

Multiple comparisons were performed using Stata 9.0 software (Stata Corp., College Station, Tex., U.S.A.). Statistical analysis was performed using the Bonferroni correction for multiple comparisons. Values of $p<0.05$ were considered statistically significant.

\section{Results}

With MR angiography, the aneurysm height was $13.75 \pm 0.05 \mathrm{~mm}$, the width was $13.27 \pm 0.10 \mathrm{~mm}$, and the neck width was $4.47 \pm 0.26 \mathrm{~mm}$. With DS angiography, the height was $14.18 \pm 0.10 \mathrm{~mm}$, the width was $13.82 \pm 0.33 \mathrm{~mm}$, and the neck width was $5.02 \pm 0.44 \mathrm{~mm}$. With CT angiography, the height was $14.10 \pm 0.22 \mathrm{~mm}$, the width was $13.75 \pm 0.58$ $\mathrm{mm}$, and the neck width was $5.98 \pm 0.09 \mathrm{~mm}$.
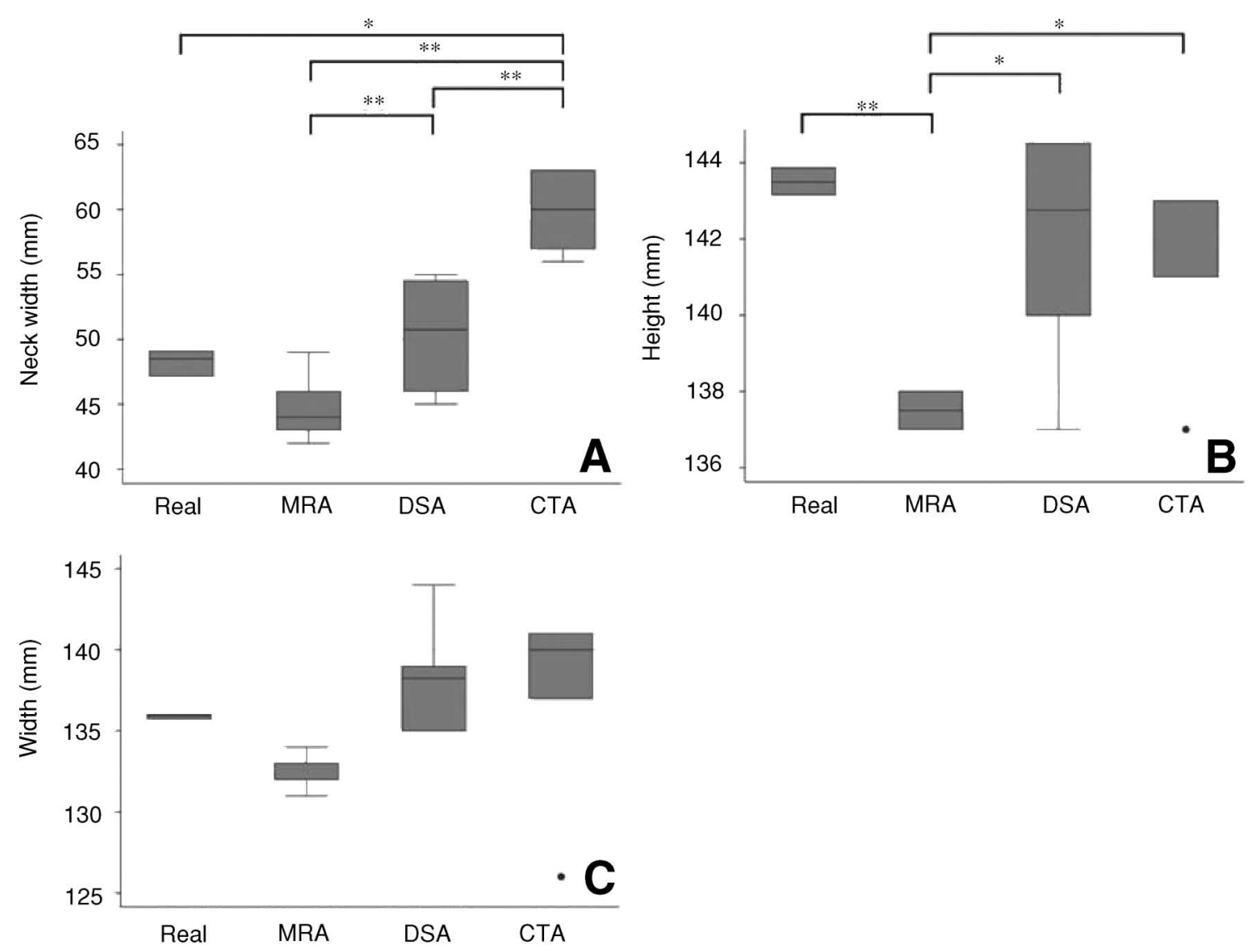

Fig. 5 Graphs showing the aneurysm measurements by each method: Real, actual measurement; MRA, magnetic resonance angiography; DSA, digital subtraction angiography; CTA, computed tomography angiography. A: Aneurysm neck width, $0.6 \mathrm{~mm}\left({ }^{*} \mathbf{p}<0.05,{ }^{* *} \mathrm{p}<0.01\right)$; B: height of aneurysm, $0.6 \mathrm{~mm}\left({ }^{*} \mathrm{p}<0.05,{ }^{*} \mathrm{p}<0.01\right)$; $\mathrm{C}$ : width of aneurysm, $0.6 \mathrm{~mm}\left({ }^{*} \mathrm{p}<0.05,{ }^{* *} \mathrm{p}<0.01\right)$. Median measurements are indicated with horizontal bars. Vertical bars indicate adjacent value ranges, and horizontal boundaries of boxes represent the first and third quartiles. The dot represents an outlier value. 
The aneurysm neck width measured on CT angiography was significantly wider than the actual value $(p<0.01)$. Furthermore, aneurysm neck widths measured on MR, DS and CT angiographies differed significantly ( $p<0.05$ each; Fig. 5A), with the measured values being highest with CT angiography, intermediate with DS angiography, and lowest with MR angiography. The aneurysm height measured under MR angiography was significantly smaller than the actual value $(\mathrm{p}<0.01)$, and was smaller than the heights measured under DS angiography $(\mathrm{p}<0.05)$ and CT angiography $(\mathrm{p}<0.05)$ (Fig. 5B). The aneurysm width measured with MR angiography tended to be smaller than the actual value. However, widths measured using the 3 modalities did not differ significantly (Fig. 5C).

\section{Discussion}

The present study confirmed that differences in clinical measurements seen occasionally in patients are also apparent under experimental conditions. CT and DS angiography yielded similar values for aneurysm height and width size. Overestimation of aneurysm neck width with CT angiography may affect treatment planning. Wide aneurysm necks require additional efforts, such as balloon-assisted techniques and intracranial stent placement, for optimal treatment results. Erroneous measurement data can influence decision making. In these circumstances, planning therapy by taking into account size-estimation tendencies for each modality is crucial.

MR angiography had the lowest spatial resolution for both slice and volume data and tended to provide widely varying results depending on the sequence and scan time used. ${ }^{16)}$ TOF, the standard angiography sequence, cannot always demonstrate the shapes of large aneurysms, due to turbulence and similar effects. Despite the low accuracy, MR angiography is the most useful screening tool because of its noninvasive nature, with no requirement for $\mathrm{x}$ ray exposure or contrast medium injection. Although MR angiography generally underestimated the aneurysm size in this study, the technique is more likely to over- or underestimate the size than DS angiography according to window adjustments.

2D/3D MR angiography measurement errors tended to increase due to the blurring components for three reasons. First, in relation to FOV, the number of matrices within an observation area is lower and the size of pixels is larger. In general, with DS, CT, and MR angiography, as pixel counts markedly differ despite the comparable imaging field sizes, "spatial resolution" varies. Large pixel size causes the partial volume effect to blur the outline of the structural margins. Also, when marked differences exist in signal intensity inside and outside structures, truncation artifacts also occur. ${ }^{23)}$ Truncation artifacts are caused by truncation of signal reading, but cannot be completely avoided, and cause additional blurring. Both the partial volume effect and truncation artifacts can be reduced by decreasing pixel sizes and increasing pixel counts. Second, MR angiography examines fluid flow, not x-ray absorption, so the error components due to shape become relatively large in fine structures with uneven flow rate distributions, such as seen in perforators. Third, the present study used a mixture of glycerol and water, not blood. Flow characteristics differ between the fluid in the present model and blood in actual clinical settings. The signal intensity of vessels as assessed by TOF MR angiography is influenced by TR, flip angle, 3D slab thickness, flow rate, and fluid $\mathrm{T}_{1}$ value. By continuing to excite at an interval (TR) shorter than the $\mathrm{T}_{1}$ value of an object, signals become saturated. Signal intensity is determined by the amount of unexcited components within a measurement volume (in-flow effect). The $\mathrm{T}_{1}$ value of physiological saline is greater than that of blood, and because the ratio of signal recovery within the same TR is low (i.e., saturation is more likely), signals themselves become weak. Therefore, the error range for size measurement for TOF MIP MR angiography is greater compared to the other modalities.

The characteristics of CT angiography are accuracy and high spatial resolution of each slice. Resolution along the longitudinal axis of the body relies on beam thickness and reconstructed slice thickness at the time of scanning. Therefore, accuracy can vary with the direction of vessels and the shapes of target objects. Although isotropic volume data must be obtained with sufficiently thin slices to achieve suitable precision, minimum slice thickness is limited by the rate of contrast medium infusion and scanning speed. An advantage of multislice CT angiography is the presence of numerous rows. Despite being somewhat invasive, with x-ray exposure and use of contrast agents, the procedure can be performed quickly without placing an excessive burden on the patient. Measured neck widths are always greater than those obtained from other techniques. With multidetector CT angiography, slice thickness typically increases with slice beam thickness and pitch (table speed), and blurring appears in the scanning direction $(\mathrm{z}$ direction) because of the partial volume effect. ${ }^{5,13)}$ Due to this partial volume effect, CT angiography cannot accurately depict the shape of an object smaller than the minimum unit volume, which is similar to slice thickness, resulting in a blurring of 

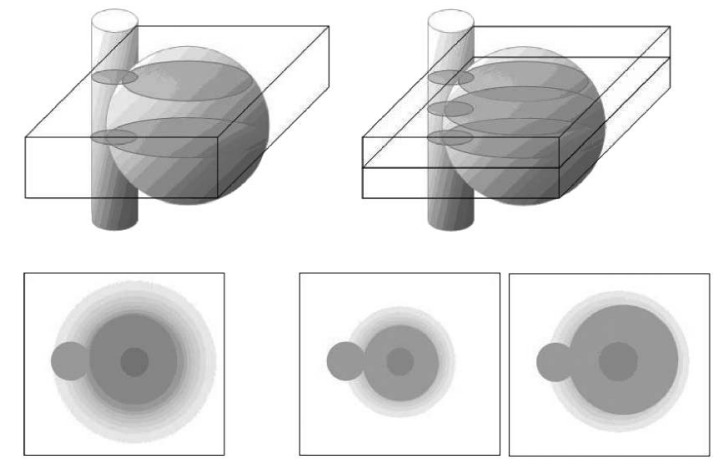

Fig. 6 Illustration of the partial volume effect. When slices are thick, images showing the vessel and aneurysm are blurred. When slices are thin, images become clearer. This partial volume effect of slice volume explains why the neck of an aneurysm looks magnified if the vessel and aneurysm surface cross obliquely.

margins, errors of object shape, and value changes. This effect is evident when the thickness of the slice is greater than that of the scanned object. Even if the slices are sufficiently thin, objects with different diameters along the axis, such as spheres, are more easily affected than objects with uniform diameters wherever sliced, such as cylinders (Fig. 6). Because of the partial volume effect, the aneurysm neck, which is located where the main vessel and the aneurysm surface cross obliquely, is presumably magnified. Therefore, neck distances measured with CT angiography were increased in this study. For the most part, CT angiography is accurate, but accuracy decreases when objects are inclined to the scanning plane.

Angiography offers the highest spatial resolution of 2D imaging of all modalities, including digital angiography and DS angiography. Angiography also offers high spatial resolution for 3D reconstructed images and can obtain evenly isotropic volume data for all xyz axis directions according to the fundamental theory of reconstruction. ${ }^{3)}$ Therefore, the accuracy is consistent regardless of vessel direction. However, angiography is the most invasive and burdensome technique for the patient, in addition to requiring x-ray exposure and contrast agent injection. The procedure is also time-consuming. Due to the large matrix, the overall precision of measurement with angiography is high. In our model of orthogonal projections to the model, we expected the best precision performance of the method in 2D imaging, with advantage over 3D imaging.

One limitation of this study was the lack of a standard protocol for window settings. With the workstation software we used, size measurements could be controlled by modifying window levels. Software using measurements from the workstation does not include any such protocol. Protocols for window levels that accurately reflect size should be provided by software developers for the respective hardware. Although we expect that the precision of each modality will continue to improve in the future, error-free measurements will be difficult to obtain without improvement of measurement technologies. The precision of each method and associated tendencies must be clarified so that the use of these techniques can be optimized.

We believe that the presence of errors in aneurysm measurement, as originating in the properties of the methodology applied, cannot be ascribed to the hardware systems used by us, but to the properties of the parameters used for image acquisition, and therefore are integral to all hardware systems using the same methodology. That means that a similar study can be performed on other types of MR imaging, CT, and angiography equipment, with similar qualitative results. In consideration of the clinical applicability of the study, every system can achieve adequate imaging suitable for aneurysm measurement and, recommendations should be available to the investigators for proper interpretation of measurement errors.

Accurate measurements of aneurysm neck and dome sizes are important for the treatment of cerebral aneurysms. The present results suggest that CT angiography overestimates aneurysm neck width. Relative values (rather than absolute values) should thus be standardized for each method, or trends in accuracy should be clarified so that real size estimation for obliteration can be planned. Adequate error tendencies and estimation data can be attached to the image processing software, providing the clinician with more realistic aneurysm measurements.

\section{Acknowledgments}

We wish to thank Mr. Akito Hayashi and Mr. Chiaki Imura of Siemens-Asahi Medical Technologies Ltd. for their helpful comments on imaging and software analysis, and Hitoshi Kuriyama, Toshihiro Takashima, Mayu Fujii, Toru Matsumoto, and Momoko Fukushima of Waseda University, Tokyo, for preparing the silicone model and circulation system.

We would also like to thank Dr. Mitsuyoshi Urashima of the Division of Clinical Research and Development, The Jikei University School of Medicine, for statistical advice and Dr. Kostadin L. Karagiozov, M.D., Ph.D., for his advice and editing the English version of the article. 


\section{References}

1) Edelman RR, Ahn SS, Chien D, Li W, Goldmann A, Mantello M, Kramer J, Kleefield J: Improved time-offlight MR angiography of the brain with magnetization transfer contrast. Radiology 184: 395-399, 1992

2) Ernemann UU, Gronewaller E, Duffner FB, Guervit $\mathrm{O}$, Claassen J, Skalej MD: Influence of geometric and hemodynamic parameters on aneurysm visualization during three-dimensional rotational angiography: an in vitro study. AJNR Am J Neuroradiol 24: 597-603, 2003

3) Feldkamp L, Davis L, Kress J: Practical cone-beam algorithm. J Opt Soc Am A 1: 612-619, 1984

4) Fernandez Zubillaga A, Guglielmi G, Vinuela F, Duckwiler GR: Endovascular occlusion of intracranial aneurysms with electrically detachable coils: correlation of aneurysm neck size and treatment results. AJNR Am J Neuroradiol 15: 815-820, 1994

5) Gupta R, Bartling SH, Basu SK, Ross WR, Becker H, Pfoh A, Brady T, Curtin HD: Experimental flat-panel high-spatial-resolution volume CT of the temporal bone. AJNR Am J Neuroradiol 25: 1417-1424, 2004

6) Harbaugh RE, Schlusselberg DS, Jeffery R, Hayden S, Cromwell LD, Pluta D, English RA: Three-dimensional computed tomographic angiography in the preoperative evaluation of cerebrovascular lesions. Neurosurgery 36: 320-327, 1995

7) Hayashi K, Nagasawa S, Naruo Y, Okumura A, Moritake K, Handa H: Mechanical properties of human cerebral arteries. Biorheology 17: 211-218, 1980

8) Kalender WA: Principles of computed tomography, Section 1, in: Computed Tomography: Fundamentals, System Technology, Image Quality, Applications. Erlangen, Publicis Corporate Publishing, 2005, pp 17-35

9) Kawasaki T, Sasayama S, Yagi S, Asakawa T, Hirai T: Non-invasive assessment of the age related changes in stiffness of major branches of the human arteries. Cardiovasc Res 21: 678-687, 1987

10) Keogh AJ, Vhora S: The usefulness of magnetic resonance angiography in surgery for intracranial aneurysms that have bled. Surg Neurol 50: 122-129, 1998

11) Masaryk AM, Frayne R, Unal O, Rappe AH, Strother CM: Utility of CT angiography and MR angiography for the follow-up of experimental aneurysms treated with stents or Guglielmi detachable coils. AJNR Am J Neuroradiol 21: 1523-1531, 2000

12) Matsumoto $M$, Sato $M$, Nakano $M$, Endo $Y$, Watanabe Y, Sasaki T, Suzuki K, Kodama N: Threedimensional computerized tomography angiographyguided surgery of acutely ruptured cerebral aneurysms. J Neurosurg 94: 718-727, 2001

13) Matsushima $M$, Adachi $T$, Tanaka R, Kikuchi $Y$, Shimoda M, Yoneda T, Yonezawa T: [Study of optimal imaging parameters for digitally reconstructed radiographs (DRR) in radiotherapy treatment planning using single-slice helical CT]. Nippon Hoshasen
Gijutsu Gakkai Zasshi 60: 528-536, 2004 (Japanese)

14) McDougall CG, Halbach VV, Dowd CF, Higashida RT, Larsen DW, Hieshima GB: Endovascular treatment of basilar tip aneurysms using electrolytically detachable coils. J Neurosurg 84: 393-399, 1996

15) Niki K, Sugawara M, Chang D, Harada A, Okada T, Sakai R, Uchida K, Tanaka R, Mumford CE: A new noninvasive measurement system for wave intensity: evaluation of carotid arterial wave intensity and reproducibility. Heart Vessels 17: 12-21, 2002

16) Ozsarlak O, Van Goethem JW, Maes M, Parizel PM: MR angiography of the intracranial vessels: technical aspects and clinical applications. Neuroradiology 46: 955-972, 2004

17) Piotin M, Gailloud P, Bidaut L, Mandai S, Muster M, Moret J, Rufenacht DA: CT angiography, MR angiography and rotational digital subtraction angiography for volumetric assessment of intracranial aneurysms. An experimental study. Neuroradiology 45: 404-409, 2003

18) Sato M: [The usefulness of Gadolinium-enhanced MR angiography for large cerebral aneurysm]. No To Shinkei 55: 542-543, 2003 (Japanese)

19) Scheel P, Ruge C, Schoning M: Flow velocity and flow volume measurements in the extracranial carotid and vertebral arteries in healthy adults: reference data and the effects of age. Ultrasound Med Biol 26: 1261-1266, 2000

20) Schievink WI: Intracranial aneurysms. N Engl J Med 336: 28-40, 1997

21) Watanabe Z, Kikuchi Y, Izaki K, Hanyu N, Lim FS, Gotou H, Koizumi J, Gotou T, Kowada M, Watanabe $\mathrm{K}$ : The usefulness of 3D MR angiography in surgery for ruptured cerebral aneurysms. Surg Neurol 55: 359-364, 2001

22) White PM, Wardlaw JM, Easton V: Can noninvasive imaging accurately depict intracranial aneurysms? A systematic review. Radiology 217: 361-370, 2000

23) Wood ML, Henkelman RM: Truncation artifacts in magnetic resonance imaging. Magn Reson Med 2: 517-526, 1985

24) Yamura M, Hirai T, Korogi Y, Ikushima I, Yamashita Y: Quantitative evaluation of measurement accuracy for three-dimensional angiography system using various phantoms. Radiat Med 23: 175-181, 2005

25) Zouaoui A, Sahel M, Marro B, Clemenceau S, Dargent N, Bitar A, Faillot T, Capelle L, Marsault C: Three-dimensional computed tomographic angiography in detection of cerebral aneurysms in acute subarachnoid hemorrhage. Neurosurgery 41: 125-130, 1997

Address reprint requests to: Hiroyuki Takao, M.D., Ph.D., Division of Endovascular Neurosurgery and Neurosurgery, Department of Neurosurgery, Jikei University School of Medicine, 3-25-8 Nishi-shinbashi, Minato-ku, Tokyo 105-8461, Japan.

e-mail: takao@jikei.ac.jp 